\title{
La diferencia sexual: ¿una ficción necesaria?
}

\section{The Sexual Difference: a Necessary Fiction?}

\begin{abstract}
RESUMEN
Por una parte, el feminismo de la diferencia señala el carácter androcéntrico del pensamiento occidental, el cual universaliza lo masculino y relega lo femenino a la otredad. Esta corriente de pensamiento construye una política feminista cuya finalidad es el reconocimiento de lo femenino partiendo de la experiencia específica de ser mujer. En consecuencia, la diferencia sexual aparece como una categoría de análisis central. Por otra parte, la teoría del género resalta el carácter heterosexual de la categoría de sexo. El presente estudio aborda dicha problemática tomando en consideración los escritos de Wittig sobre el pensamiento heterosexual y su influencia en el pensamiento de Butler, así como la obra de Braidotti, una de las principales teóricas de la diferencia sexual. El objetivo será clarificar la tensión entre la teoría del género y el pensamiento de la diferencia sexual con el fin de encontrar una estrategia que evite la exclusión.
\end{abstract}

Palabras clave: feminismo, diferencia sexual, género, pensamiento heterosexual.

\begin{abstract}
On the one hand, the difference feminism points out the androcentric character of the Western thought which universalizes the masculine and relegates the feminine to otherness. This school of thought makes feminist politics whose aim is the recognition of the feminine considering the specific experience of being a woman. As a consequence, the sexual difference appears as a central analytic category. On the other hand, gender theory remarks the heterosexual implication of the category of sex. This research deals with that subject taking into account Wittig's writings about the straight mind and its influence on Butler's thought, as well as Braidotti's work, one of the main theorists of sexual difference. The objective will be to clarify the tension between gender theory and the thought of sexual difference in order to find a strategy that would avoid exclusion.
\end{abstract}

Keywords: Feminism, Sexual Difference, Gender, Straight Mind.

\section{SUMARIO}

1.Introducción. 2. Posiciones políticas en torno a las categorías «sexo» y «género». 3. Conclusiones. 4. Bibliografía.

1 Universidad Complutense de Madrid, anavelascolazaro@gmail.com 


\section{Introducción}

La diferencia sexual es una categoría cuya relevancia histórica, política y social no puede ponerse en duda. Por ello, cabe señalar que el título del presente trabajo no obedece a una pretensión de negar los efectos que produce la diferencia sexual en la vida de las personas. La finalidad es analizar las implicaciones de dicha categoría y su provecho estratégico para la teoría feminista.

Para comenzar un rápido estudio del paradigma de la diferencia sexual necesariamente nos enfrentamos a asuntos tales como la significación del cuerpo, la función simbólica del lenguaje y la dimensión sociológica de la subjetividad. Son precisamente estos temas de investigación los que podemos situar en el corazón de dos teorías que van a aportar debates muy productivos en el pensamiento feminista: la teoría de la diferencia sexual y la teoría del género. Judith Butler y Rosi Braidotti han contribuido a esa producción intelectual con valiosas reflexiones. Butler, una de las autoras más relevantes de la teoría del género, ha revisado el uso de la categoría de la diferencia sexual en la teoría feminista con el objetivo de denunciar el esquema heterosexual sobre el que se funda. Braidotti, considerada una pensadora de segunda generación de la diferencia sexual, ha encaminado sus esfuerzos a repensar el sujeto político del feminismo de forma que dé cuenta de la experiencia corporal de ser mujer. Ambas autoras han dialogado de forma enriquecedora sobre estas cuestiones en una entrevista que Butler realizó a Braidotti².

\section{Posiciones políticas en torno a las categorías «sexo» y «género»}

Generalmente la diferencia sexual ha sido entendida como la base biológica y el género como una construcción cultural. Siguiendo esta caracterización, la diferencia sexual aparece como un reducto natural y ahistórico que supone la solidificación de la oposición binaria de los sexos. El concepto de género emerge en el pensamiento feminista con el fin de separar las construcciones sociales y culturales de lo biológico, esto es, señalar los rasgos y patrones de comportamiento considerados femeninos como una asimilación social por parte del individuo y no como una derivación natural de la diferencia sexual (Lamas, 1999: 147). En este sentido, el género supone una categoría de análisis muy provechosa para la teoría feminista puesto que implica enfrentarse al discurso patriarcal del determinismo biológico. Sin embargo, la categoría de género conlleva algunos problemas para la investigación feminista ya que surge en el contexto norteamericano con el término anglosajón gender y éste no se corresponde con su traducción en otras lenguas. En inglés hace referencia a los sexos, pero en español, por citar un ejemplo, indica la especie o clase a la que pertenecen las cosas. Siguiendo la definición de la Real Academia de la Lengua Española, «género» sólo hace referencia a los sexos en la acepción

2 Rosi Braidotti with Judith Butler (1994) «Feminism by Any Other Name» en Differences: A Journal Of Feminist Cultural Studies, Vol. 6, n 2-3. pp. 29-61. En castellano: «El feminismo con cualquier otro nombre» en Rosi Braidotti, Feminismo, diferencia sexual y subjetividad nómade. Barcelona: Gedisa, 2004. pp. 69-106 
gramatical del término. Esta dificultad de traducción ha tenido como consecuencia que en el pensamiento feminista europeo, concretamente en los países de lenguas romances, haya sido más común hablar de estudios de las mujeres que de estudios de género.

Las corrientes de pensamiento feminista que han problematizado la identidad de género, tomando en consideración las prácticas sexuales, han denunciado acertadamente que centrarse en la diferencia sexual como rasgo principal de análisis supone reforzar el sistema binario de los sexos y, con ello, la normatividad heterosexual. Un estudio lúcido de la categoría de sexo y de sus implicaciones aparece en 1992 con la publicación de The Straight Mind and Other Essays de la mano de la escritora y teórica francesa Monique Wittig. Consiste en una recopilación de artículos escritos desde finales de los años 70 hasta aproximadamente 1990 donde se trata de denunciar la heterosexualidad imperante en el pensamiento occidental.

Según Wittig, la categoría de sexo sostiene un orden social, económico, político e ideológico en el que las mujeres aparecen como esclavas de los hombres y los individuos son sometidos a un sistema económico heterosexual. El discurso de la diferencia sexual es aquél con el que se perpetúa ese orden, presentando como natural el binomio hombre/mujer - masculino/femenino. Lo que Wittig denomina el pensamiento heterosexual comprende un conglomerado de todo tipo de disciplinas, teorías e ideas preconcebidas basadas en conceptos como mujer, hombre o diferencia que desarrolla una interpretación totalizadora de la historia, de la realidad social, de la cultura y del lenguaje al mismo tiempo. Se produce la diferencia entre los sexos como dogma político y filosófico, y se establece como obligatoria la relación social entre el hombre y la mujer. Este pensamiento hace uso de unas categorías que en realidad solo pueden tener un sentido absoluto en la heterosexualidad. En consecuencia, la homosexualidad no puede ser concebida (Wittig, 1992: 28).

Rechazando conceptos tales como mujer $u$ hombre -ya que mantienen el régimen heterosexual-, el sujeto de resistencia se va a construir a partir de la figura de la lesbiana. Dado que las lesbianas no viven bajo las condiciones del régimen -rechazo al coito y a vincularse social y emocionalmente con hombres, lo que significa dentro de la economía heterosexual el rechazo a ser esclavas-, no pueden ser concebidas bajo los parámetros de la diferencia sexual. Consecuentemente con esta argumentación, Wittig sostenía que las lesbianas no son mujeres.

Su obra supuso un análisis que ayudó a comprender mejor las implicaciones teóricas y políticas del discurso de la diferencia sexual. Judith Butler (1990a) recoge el trabajo de Wittig en su indagación de la relación entre el lenguaje y el poder con el propósito de denunciar la heteronormatividad presente en el feminismo, el cual mantenía relativamente indemne la facticidad de la «mujer» en el centro de sus debates. En contraposición a esto, Butler defiende la naturaleza provisional de la identidad, aunque afirmará al mismo tiempo -asumiendo el desafío que esto entraña- que la identidad es una necesidad política con una base empírica (Kirby, 2011: 36). Butler reconoce los logros teóricos llevados a cabo por Wittig al identificar la diferencia sexual como «un acto interpretativo cargado de supuestos normativos sobre un sistema de género binario» (Butler, 1990b: 202). En este sentido, aunque 
se va a distanciar del pensamiento de Wittig al considerar que ésta mantiene los presupuestos del humanismo ${ }^{3}$, va a tomar de su obra la no naturalidad del sexo, es decir, la condición de ficción política de la diferencia sexual. Para la pensadora estadounidense, "comprender el género como una categoría histórica es aceptar que el género, entendido como una forma cultural de configurar el cuerpo, está abierto a su continua reforma, y que la "anatomía" y el "sexo" no existen sin un marco cultural» (Butler, 2006: 25). En otras palabras, el sexo también está sujeto a la interpretación cultural. No se trata de negar la existencia de las distinciones anatómicas, sino de poner de relieve y cuestionar el régimen social que, a partir de un sistema binario, interpreta las partes sexuales que ayudan en el proceso de reproducción como significantes que establecerán la identidad sexual y determinarán la vida del sujeto. Butler, siguiendo a Wittig, lo ilustra así:

[Wittig] señala que existen otros tipos de diferencias entre la gente, diferencias de: forma y tamaño, de formación del lóbulo de las orejas y el tamaño de la nariz, y no preguntamos qué clase de lóbulo de orejas tiene. Preguntamos inmediatamente por determinados rasgos anatómicos sexualmente diferenciados porque suponemos que esos rasgos de algún modo determinarán el destino social de ese bebé, y ese destino, cualquiera que sea, es estructurado por un sistema de género predicado sobre la naturalidad de las oposiciones binarias y, en consecuencia, de la heterosexualidad. (Butler, 1990b: 203)

Según Butler, El pensamiento heterosexual supuso un paso adelante en la carrera por el rechazo al determinismo biológico que inauguró Simone de Beauvoir con la máxima: «no se nace mujer: llega una a serlo» (Beauvoir, 1987: 13). Wittig fue más lejos al determinar que el sexo es una unidad ficticia y, en coherencia, que la distinción entre sexo y género no es operativa, ya que ambas nociones son resultado de construcciones socioculturales y lingüísticas. Butler recoge esta idea y reformula los textos de la escritora francesa al afirmar que la diferencia sexual es creada al ser nombrada ${ }^{4}$. Así, toma la obra de Wittig como apoyo a sus reflexiones sobre la performatividad lingüística que en Wittig solo están sugeridas. En los textos de Butler, el género es definido explícitamente como un acto performativo (Burgos, 2003: 27). Esta concepción del género tuvo una gran acogida dentro del marco de los llamados «estudios queer», una disciplina académica que cuestiona todo esencialismo y propone la deconstrucción de la identidad sexual y de género. La práctica de la performatividad subversiva que presenta Butler permite desestabilizar los roles de género que, tal y como señaló Wittig, se sustentan en un pensamiento heterosexual. El hecho de que pueda tener lugar ese acto de deconstrucción de la identidad probaría aquello que afirma la teoría queer: la contingencia de la propia identidad.

Por otra parte, el paradigma de la diferencia sexual ha pensado esta categoría desde una perspectiva feminista. Con origen en Europa, esta corriente surge como

3 Butler dice así: «Wittig concibe una sociedad sin sexo, argumentando que el sexo, al igual que la clase, es un constructo que debe ser depuesto inevitablemente. El programa de Wittig parece, desde luego, profundamente humanista en su exigencia de la erradicación del sexo» (Butler, 1990b: 204).

4 Butler escribe: «Para Wittig, cuando nombramos la diferencia sexual, la creamos» (Butler, 1990b: 202). 
un esfuerzo de pensar la especificidad de las mujeres fuera del marco patriarcal, donde lo femenino aparece siempre como negatividad. Se trata, por el contrario, de pensar desde la positividad de lo diferente. Aquí los conceptos como sexo o diferencia juegan un papel central puesto que es la experiencia de ser mujer la base en la que se apoyan las reivindicaciones políticas. Estas teóricas van a aportar una reflexión necesaria sobre la condición marginal de lo femenino en una sociedad donde imperan los valores masculinos.

Surge también un rechazo a concebir dicha diferencia en términos esencialistas. El pensamiento de Rosi Braidotti parte de una concepción inmanente del sujeto, el cual tiene como rasgo principal la capacidad de ser afectado y de afectar. La subjetividad siempre se va a formar a partir del arraigo local del cuerpo, de sus experiencias y su anhelo de devenir. Esto rompe con el principio básico de la modernidad: el sujeto cognoscente, universal y neutro del cartesianismo. Según Braidotti, todo pensamiento está localizado y codificado culturalmente. La enunciación de una instancia filosófica se asienta en un momento prefilosófico, a saber: la capacidad humana de tener predisposición, receptividad y deseo hacia el pensar. Esta capacidad introduce al sujeto en una red de discursividad, lenguaje y poder (Braidotti, 2004: 41-42). El deseo es la condición a priori del pensamiento de manera que no puede dar cuenta de él. Esto cuestiona un rasgo cultural claramente moderno: el de la neutralidad de las ciencias, dado que señala el papel del lenguaje en la elaboración de todo sistema de conocimiento. En todo proceso de pensamiento hay rasgos afectivos. El deseo es la base que sustenta el lenguaje y por ello es algo difícilmente expresable.

Braidotti presenta el cuerpo como un espacio unificado de intersección de lo biológico, lo social y lo lingüístico -entendiendo lo lingüístico como lo simbólico dentro de una determinada cultura. No es una entidad abstracta, sino materia incardinada, lo que supone que está siempre situado de manera primaria. La experiencia derivada de ser corporal es siempre sexuada dado que el sujeto siempre es generizado en las estructuras del lenguaje (siempre es «yo-ella» o «yo-él»). Sin embargo, el sujeto mujer no es una esencia nominal, sino una ficción conveniente. No es una esencia monolítica, sino un lugar de intersección de diversas experiencias en constante cambio. Por ello, la mujer habla como si fuera mujer y no lo es en sentido esencial. La ficción del «yo-ella» es necesaria para el mantenimiento de una unidad posicional de las múltiples variables que forman al sujeto. Esta ficción, en la que se apoyará la conciencia feminista, es el sujeto feminista femenino, el cual se aferra a su yo incardinado, pero sólo en la medida en que otras mujeres definen la subjetividad femenina desde sus aspectos prácticos. El «yo-mujer» no consiste en una identidad preexistente.

Braidotti considera importante distinguir la subjetividad de la identidad: la subjetividad política se elabora de manera deliberada pero la identidad tiene que ver con procesos inconscientes. Considera que la política feminista debe ser reformulada de manera que tenga también en cuenta los deseos inconscientes que forman la experiencia de las mujeres. Según Braidotti, el deseo siempre está ligado a las diferentes experiencias corporales, de ahí que haya tantas identidades 
como diferentes experiencias (la formación de identidades tales como «mujer», «chicana» o «lesbiana» son resultado de una determinada situación). La subjetividad que reivindica Braidotti para el feminismo no se articula desde una concepción nostálgica de lo que sería auténticamente la identidad femenina, sino que se da un salto ontológico hacia delante. Este salto es el colectivo de las mujeres construyendo su propia identidad, es decir, una representación de lo que implica ser mujer aquí y ahora con una fuerza política positiva. Es la conciencia resultante de la mezcla del deseo ontológico de ser con un movimiento político colectivo. La comunidad política de las mujeres es, por tanto, muy importante para la formación del sujeto feminista femenino.

Para Braidotti, la diferencia sexual no es un concepto ahistórico, monolítico, alejado de las prácticas y dogmático, sino que se basa en la experiencia corporal de la mujer. Por ello la subjetividad es múltiple, tanto como las diferencias entre las mujeres. Se concibe lo simbólico de una manera dinámica e histórica, dado que se da de forma simultánea con lo social. Por este motivo, Braidotti insiste en que su concepción del sujeto recoge las diferentes variables del mismo.

No obstante, es precisamente en la manera de entender lo simbólico y lo social donde Butler se va a distanciar de manera aguda. Para la pensadora estadounidense, hay cierta ambigüedad e, incluso, contradicción, en la defensa de Braidotti del dinamismo e historicidad de lo simbólico en tanto que al mismo tiempo distingue lo simbólico de lo social y lo material (Burgos, 2008: 348). En el intercambio comunicativo que mantuvieron ambas autoras en 1994, Butler sostiene que afirmar la simultaneidad de lo simbólico y lo social es también afirmar la separabilidad. Braidotti insistirá en que lo simbólico no es entendido como una estructura ahistórica, sino como un proceso dinámico de producción de prácticas significantes donde se entreteje las condiciones lingüísticas y sociales de esta producción. Sin embargo, Butler encuentra en esta noción de lo simbólico una idealización de lo social y, como consecuencia, denuncia su conexión con el poder:

Me pregunto si lo simbólico está destinado a operar de esa forma, vale decir, como un conjunto de estructuras y dinámicas reguladoras que ponen un límite a lo que puede y no puede ser elegido. ¿Quién, por ejemplo, es el autor que decide estas cuestiones y por qué la autoría misma está decidida de antemano precisamente por este funcionamiento simbólico? Pienso que lo simbólico designa el carácter idealizado del poder regulador y que ese poder debe situarse y criticarse, en última instancia, dentro de una concepción intensificada de lo social. Se trata, pues, de una clara diferencia entre nosotras (Butler en Braidotti, 2004: 102)

El proyecto que persigue la teoría del género de Butler consiste en plantearse de qué manera se puede reconfigurar la relación entre lo simbólico y lo social, con el fin de posibilitar que desde lo social se lleve a cabo una alteración de lo simbólico (Burgos, 2005: 719). Para Butler, entender la diferencia sexual como simbólica sería también una manera de reforzar un sistema binario de género y legitimar una organización dada de heterosexualidad obligatoria. 
Sin embargo, Braidotti considera que, aunque estamos ante dos marcos conceptuales diferentes con reclamos opuestos (por un lado, las teóricas de la diferencia sexual ponen el énfasis en el empoderamiento del sujeto feminista femenino y, por otro lado, las teóricas del género conciben lo femenino como un embrollo metafísico que es mejor descartar), estos reclamos «no constituyen una división entre la heterosexualidad y la teoría lesbiana -es decir, una diferencia sexual acotada por la heterosexualidad y un género desplazado hacia una teoría lesbiana- sino, más bien, un disenso dentro de las teorías y prácticas de la homosexualidad femenina» (Braidotti, 2004: 92). Según Braidotti, el lesbianismo está en el corazón del pensamiento de la diferencia sexual ya que algunas teóricas como «Cixous o Irigaray postulan el deseo lesbiano en un continuum con la sexualidad femenina, especialmente con el apego a la madre» (Braidotti, 2004: 92). Esto, sin embargo, no lo puede sostener Butler ya que el deseo lesbiano se entrecruza con normas y figuras masculinas y, por lo tanto, el discurso de la especificidad femenina para el lesbianismo es insuficiente. Lo masculino y lo femenino no pueden ir ligados a una corporalidad específica. Siguiendo la crítica de Wittig a la categoría de sexo, ésta es una construcción social, política e ideológica del pensamiento heterosexual. Según Butler, el género es el producto de la repetición de la norma, un imaginario que moldea también el cuerpo. Sin embargo, el sujeto no acata siempre de manera fiel la norma, lo que supone sobrepasar los intereses del poder -como sería, por ejemplo, la reapropiación de la masculinidad por parte de las lesbianas (Butler, 2011). Por lo tanto, la diferencia sexual como anclaje del discurso, ya sea biológica, social o simbólica, es un presupuesto incompatible con la teoría queer. Por otra parte, para Braidotti el olvido de la diferencia sexual trae implícito el riesgo de la posible homologación de lo femenino al modelo de la masculinidad. Por ello, es importante mantener el marco de referencia del dimorfismo sexual, ya que trae a debate la realidad cultural y política de la dominación patriarcal (Femenías y Ruíz, 2004: 9).

A partir de esta tensión se puede observar que la focalización en la diferencia sexual supone negar u oscurecer la asimetría de las relaciones homo/hetero, así como el giro al género oscurece o niega la relación asimétrica entre los sexos (Braidotti, 2004: 94). Esta ambivalencia supone las respectivas acusaciones entre Braidotti y Butler.

\section{Conclusiones}

Al rechazar el status ahistórico y natural de la categoría de sexo, aparece un escenario que posibilita identificar la unidad de coherencia sexo-género impuesta por el régimen heterosexual como una ficción política que obedece a los intereses de perpetuar su dominación. Incluso cuando se concibe la realidad de la diferencia sexual por su carácter simbólico, caben las sospechas de no ser más que una abstracción de su significado social y, por tanto, una estrategia para asignarle un status pre-social a un discurso construido socialmente.

Por otra parte, las teóricas de la diferencia sexual resaltan, con acierto, el hecho de que el sujeto masculino ha sido universalizado por parte de la razón occidental, 
lo que tiene como consecuencia considerar a lo masculino como lo específicamente humano y racional, y a lo femenino como la otredad. En oposición a este esquema patriarcal, el feminismo de la diferencia sexual ha tratado de reivindicar el valor propio de lo femenino. Esto se ha traducido en determinadas prácticas, como la reclamación del reconocimiento de los cuidados, trabajo que tradicionalmente es desempeñado por mujeres y que ha permanecido -y aún permanece en gran medidaen la invisibilidad de la esfera privada. La lucha por la igualdad ha reivindicado la posibilidad de las mujeres de acceder al ámbito de lo público, siendo dicho acceso un elemento clave a seguir en la agenda política. Sin embargo, para las pensadoras de la diferencia esta estrategia política -la focalización en el libre acceso de las mujeres a espacios masculinizados en detrimento de la reivindicación por el reconocimiento de lo femenino- conlleva el mantenimiento del falogocentrismo, es decir, la metafísica occidental donde la razón masculina aparece como lo Uno. En este sentido, la categoría de la diferencia sexual sirve para elaborar una imprescindible crítica para la construcción de un proyecto político sin la violencia que supone la imposición de unos parámetros valorativos culturales de carácter androcéntrico.

Aceptando la utilidad de la noción de la diferencia sexual para el análisis feminista y, por otra parte, las dificultades que encontramos en la misma como reforzamiento del género binario $y$, con ello, del régimen heterosexual, cabe preguntarse de qué manera puede la teoría feminista sacar provecho de dicha categoría.

El cuerpo femenino al que apelan las teóricas de la diferencia sexual es el mismo que el activismo queer parodia. Como consecuencia, la problematización del género tiene, en algunos aspectos, una relación difícil con el feminismo. No obstante, esta difícil relación no debe conducirnos a considerar ambas teorías y prácticas como necesariamente enfrentadas. Tanto el pensamiento de la diferencia sexual como la teoría del género aportan visiones que enriquecen y fortalecen diferentes estrategias de resistencia para las mujeres. Establecer una jerarquía entre las diferentes teorías críticas por su poder explicativo supondría reconocer una mayor urgencia a un determinado cambio social sobre otros, lo que sería cometer el error de la exclusión.

\section{BIBLIOGRAFÍA}

BeAuvoir, Simone de (1987): El segundo sexo. Vol. 2, La experiencia vivida, Buenos Aires: Siglo XXI.

Braidotti, Rosi (2004): Feminismo, diferencia sexual y subjetividad nómade, Barcelona: Gedisa. (Traducción de Gabriela Ventureira, excepto capítulo 8: María Luisa Femenías)

BRAIDOTTI, Rosi (2005): Metamorfosis. Hacia una teoría materialista del devenir. Traducción de Ana Varela Mateos, Madrid: Akal.

Burgos, Elvira (2003): «El pensamiento de Monique Wittig y su presencia en la teoría de Judith Butler» en Thémata. Revista de Filosofía, $\mathrm{N}^{\circ} 31$, pp. 15-31.

_ (2005): «Conflicto de paradigmas: "género" y "diferencia sexual"» en Thémata. Revista de Filosofía, No 35, pp. 713-720. 
(2008): Qué cuenta como una vida. La pregunta por la libertad en Judith Butler, Madrid: Antonio Machado Libros.

ButLer, Judith (1990a): Gender Trouble. Feminism and the Subversion of Identity, New York and London: Routledge.

_ (1990b): «Variaciones sobre sexo y género. Beauvoir, Wittig y Foucault» en Teoría feminista y teoría crítica: ensayos sobre la politica de género en las sociedades del capitalismo tardío, Valencia: Ed. Alfons el Magnànim: Institució Valenciana d'Estudis i Investigació, pp. 193-211

_ (2006): Deshacer el género. Traducción de Patrícia Soley-Beltran, Barcelona: Paidós.

(2011): Mecanismos psíquicos del poder, Madrid: Ediciones Cátedra.

FEMENíAs, M ${ }^{\text {a }}$ Luisa y M Ma dos Ángeles RUÍZ (2004): «Rosi Braidotti: de la diferencia sexual a la condición nómade» en Revista Escuela de Historia, año 3, Vol. 1, N ${ }^{\circ}$ 3. http:/ / www.redalyc.org/pdf/638/63810305.pdf

KIRBY, Vicki (2011): Judith Butler: pensamiento en acción. Traducción por Diego Luis Sanromán Peña: Barcelona, Bellaterra

LAmAs, Marta (1999): «Usos, dificultades y posibilidades de la categoría género», en Papeles de Población, Vol. 5, N ${ }^{\mathrm{O}}$ 21, julio-septiembre, Universidad Autónoma del Estado de México Toluca, México, pp. 147-178.

Wittig, Monique (1992): The Straight Mind and other essays, Boston: Beacon Press.

Recibido el 31 de agosto de 2016 Aceptado el 1 de febrero de 2017 BIBLID [1132-8231 (2018): 65-73] 